\title{
A Bandpass Filter Based on Half Mode Substrate Integrated Waveguide-to-Defected Ground Structure Cells
}

\author{
Yong Mao Huang, Zhenhai Shao, Zhaosheng He, Chang Jiang You, and Di Jiang \\ School of Communication and Information Engineering, University of Electronics Science and Technology of China, \\ Chengdu 611731, China
}

Correspondence should be addressed to Zhenhai Shao; shao_zh@uestc.edu.cn

Received 22 March 2014; Accepted 14 August 2014

Academic Editor: Francisco Falcone

Copyright (C) 2015 Yong Mao Huang et al. This is an open access article distributed under the Creative Commons Attribution License, which permits unrestricted use, distribution, and reproduction in any medium, provided the original work is properly cited.

\begin{abstract}
A half mode substrate integrated waveguide-to-defected ground structure (HMSIW-DGS) cell and its embedded form are proposed to miniaturize a bandpass filter. Both cells can purchase wideband frequency response and low insertion loss, as well as simple and easy fabrication. By cascading two of them according to design requirement, an X-band bandpass filter is designed and measured to meet compact size, low insertion loss, good return loss, second harmonic suppression, and linear phase.
\end{abstract}

\section{Introduction}

In the past decade, substrate integrated waveguide (SIW) has attracted plenty of attention and has been utilized in different microwave and millimeter wave components design due to its merits of higher quality factor, low cost, high power handling capability, and easy integration [1-6]. And its related designs have been expended to on-chip scale [7]. The half mode substrate integrated waveguide (HMSIW), which not only keeps the good performance of SIW but reduces to half size or so of the SIW, has been developed and widely used in varied applications [8-10]. However, its physical size is not small enough compared with the microstrip circuitry, since its conventional rectangular cavity resonator is too large.

The defected ground structure (DGS), which is etched in the ground plane of planar circuit board, provides a promising approach to increase stopband reduction and reduce size of passive components [11-13]. In [14], a new sizereduced lowpass filter (LPF) design method was introduced, which utilized DGS and wide microstrip line to realize the shunt capacitors. Therefore, open stubs, junction elements, and very high-impedance lines, which are always adopted in conventional LPFs, are not required in the new DGS-LPF design in order to achieve compact size. However, all referred DGS above are etched into the bottom side of printed circuits board, which means that the circuits and components must be hung in the air so that they are quite inconvenient to be integrated in system. In order to keep out this drawback, one solution is to utilize DGS in coplanar waveguide (CPW) and SIW $[15,16]$, called "coplanar DGS," in order to keep the signal transmission layer and the ground layer with DGS on the same plane.

As unique DGS shapes, the complementary split ring resonator (CSRR) $[17,18]$ and the complementary spiral resonator (CSR) [19], both of which were proposed as metamaterial resonators and exhibiting negative permittivity, have been utilized in SIW successfully. Both the CSRR and CSR resonators allow the implementation of a forward-wave passband propagating below the characteristic cutoff frequency of SIW. However, insertion losses of all experimental filters reported in $[18,19]$ are not low enough, which will be unacceptable for millimeter wave applications with critical power requirement.

In this paper, a novel HMSIW-DGS cell with a simple DGS shape and its embedded form, which can also be used as a cell, are proposed. By utilizing these two kinds of cells, a three-pole cascade bandpass filter is realized. According to its measured results, low insertion loss, good return loss, compact size, and suppressed second harmonic are achieved. 


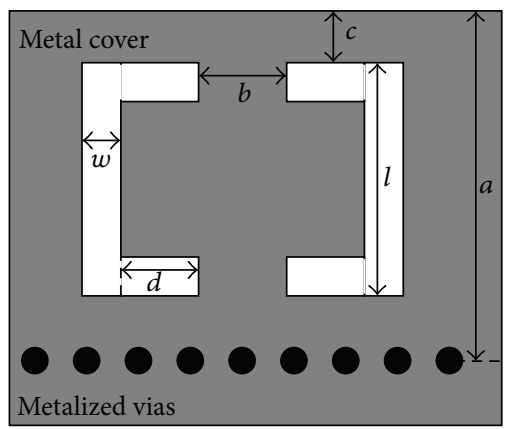

(a)

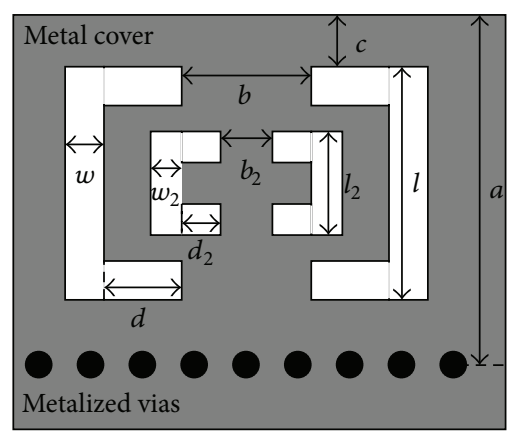

(b)

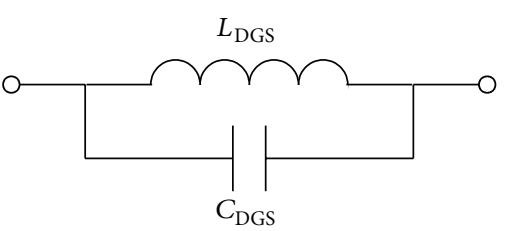

(c)

Figure 1: Proposed HMSIW-DGS cell, its embedded form, and equivalent circuit. (a) Proposed cell, (b) embedded form, and (c) equivalent circuit.

\section{Analysis and Design}

2.1. HMSIW-DGS Cells. Proposed HMSIW-DGS cell and its embedded form are shown in Figures 1(a) and 1(b), respectively. As depicted in Figures 1(a) and 1(b), since all slots are etched into the top metal cover of HMSIW, the device need not be hung in the air and can be integrated easily in the system.

To simplify the analysis, the bandpass transmission characteristic of proposed HMSIW-DGS cell can be seen as a combination of the inherent highpass characteristic provided by HMSIW and the bandgap function created by DGS. Whichever for SIW and HMSIW, as discussed in [1-3], their characteristic cutoff frequencies are mainly controlled by their widths, as labeled as " $2 a$ " and " $a$ " in Figure 2. A SIW with $2 a$ is equivalent, in terms of characteristic cutoff frequency, to a conventional rectangular waveguide with a width $a_{\text {EFF_SIW }}$ when $2 a$ and $a_{\text {EFF_SIW }}$ satisfy the fitted equation [20]

$$
a_{\mathrm{EFF} \_\mathrm{SIW}}=2 a-1.08 \frac{(2 R)^{2}}{D}+0.1 \frac{(2 R)^{2}}{2 a}
$$

where $R$ and $D$ are the vias' radius and the spacing between two adjacent vias, respectively. This equivalent rectangular waveguide has the same height $h$ and is filled with the same dielectric material as the SIW. Cutting the SIW with a width of $2 a$ in half along its longitudinal symmetry plane, an HMSIW with a width of $a$ is obtained [21], as shown in Figure 2. Similarly, its corresponding equivalent waveguide can be described as an open aperture in one side and an electric wall in the other side with a width as

$$
a_{\mathrm{EFF} \_ \text {HMSIW }}^{\prime}=\frac{a_{\mathrm{EFF} \_\mathrm{SIW}}}{2} ;
$$

owing to the fringing fields effect, this corresponding equivalent waveguide is not proper for calculating the characteristic cutoff frequency of the HMSIW. To solve this problem, a magnetic wall is used to replace the open aperture, so that an additional width, accounting for the effect of the fringing fields, should be considered to the width:

$$
a_{\text {EFF_HMSIW }}=a_{\text {EFF_HMSIW }}^{\prime}+\Delta a \text {; }
$$

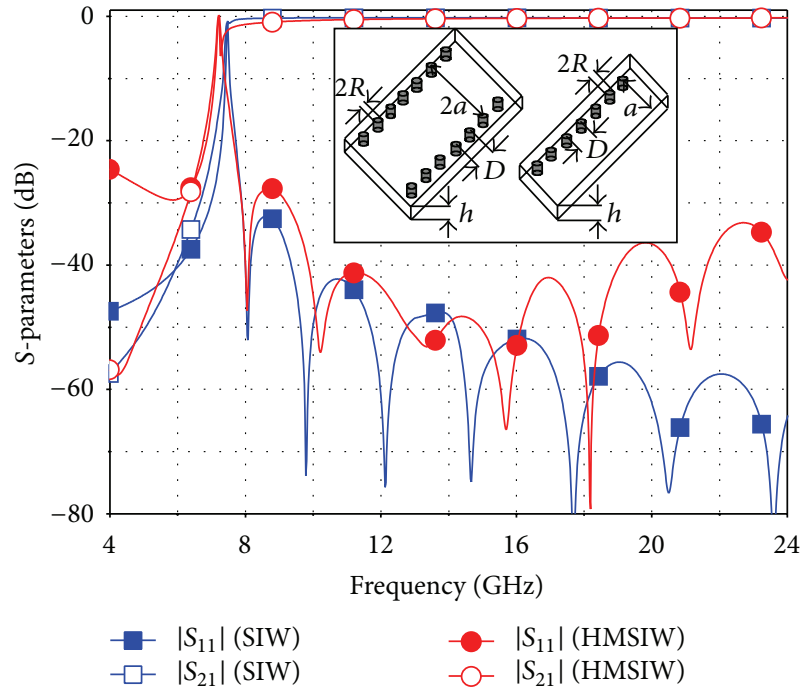

FIgURE 2: Comparison of cutoff frequencies and transmission characteristics of SIW and HMSIW. The geometrical parameters are $a=7 \mathrm{~mm}, 2 R=0.5 \mathrm{~mm}, D=0.8 \mathrm{~mm}$, and $h=0.254 \mathrm{~mm}$ and relative permittivity $\varepsilon_{r}=2.2$.

using $a_{\text {EFF_HMSIW }}$, it will be much easier to calculate the characteristic cutoff frequency of HMSIW.

From (1) to (3), it can be obtained that the characteristic cutoff frequency of an HMSIW with $a$ is lower than the characteristic cutoff frequency of a SIW with $2 a$, due to the influence of $\Delta a$. To verify this analysis, a comparison of the cutoff frequencies and transmission characteristics of a SIW and an HMSIW, with widths of $2 a$ and $a$, respectively, is given. According to the simulated results in Figure 2, the characteristic cutoff frequency of HMSIW is lower than that of SIW, and around the cutoff frequencies the insertion loss of HMSIW is higher than that of SIW, which are simulated by a commercial finite element method (FEM) simulator.

As depicted in [11-14], the DGS can be represented by an LC equivalent circuit, and the bandgap function created by the DGS can be explained by the equivalent circuit analysis theory. As shown in Figure 1(c), the equivalent circuit of the 


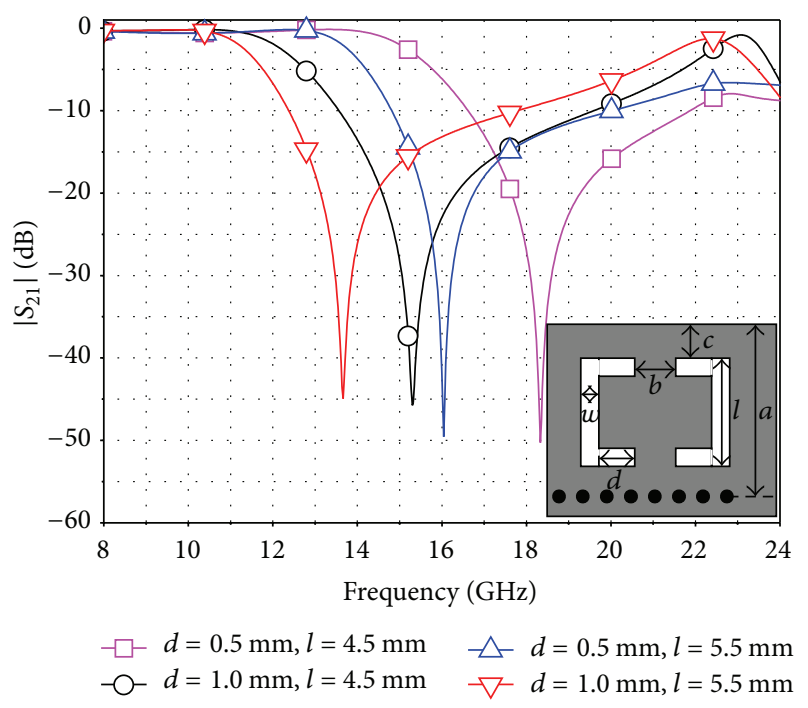

FIGURE 3: Simulated results of proposed HMSIW-DGS cell with different " $d$ " and "l." Other geometrical parameters are $a=7 \mathrm{~mm}$, $c=1 \mathrm{~mm}, w=0.5 \mathrm{~mm}$, and $b=1 \mathrm{~mm}$.

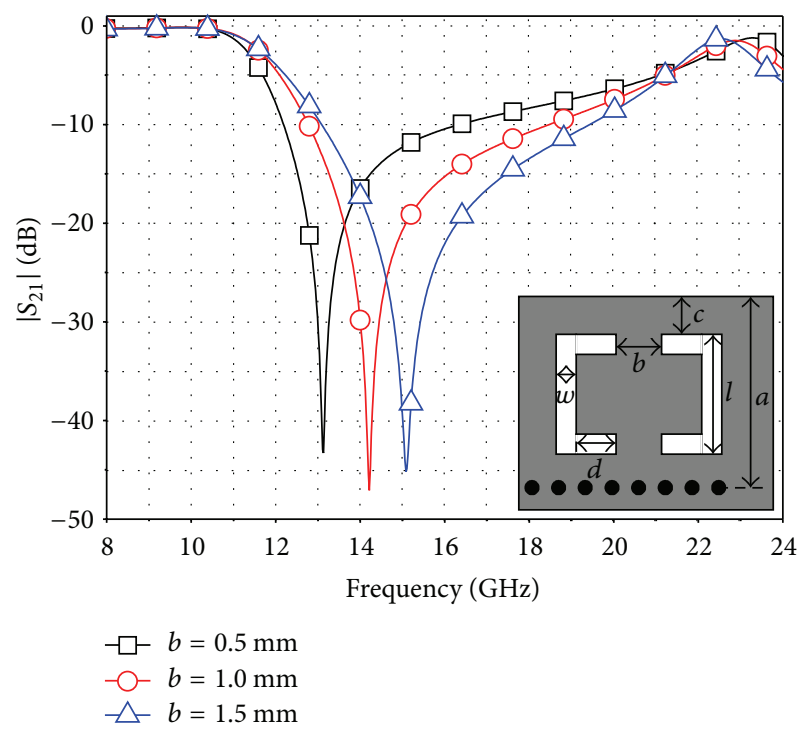

FIGURE 4: Simulated results of proposed HMSIW-DGS cell with different " $b$." Other geometrical parameters are $a=7 \mathrm{~mm}, c=$ $1 \mathrm{~mm}, w=0.5 \mathrm{~mm}, d=1 \mathrm{~mm}$, and $l=4.5 \mathrm{~mm}$.

DGS consists of a parallel inductance $L_{D G S}$ and a parallel capacitance $C_{\mathrm{DGS}}$. Their values can be computed by

$$
\begin{aligned}
C_{\mathrm{DGS}} & =\frac{5 f_{c}}{\pi\left(f_{\mathrm{bg}}^{2}-f_{c}^{2}\right)} \quad \mathrm{pF}, \\
L_{\mathrm{DGS}} & =\frac{25}{C_{\mathrm{DGS}}\left(\pi f_{\mathrm{bg}}\right)^{2}} \mathrm{nH},
\end{aligned}
$$

where $f_{c}$ and $f_{\mathrm{bg}}$, both in gigahertz, are the $3 \mathrm{~dB}$ cutoff frequency and bandgap point frequency of the DGS [13].
To make the transmission behavior and working principles of proposed HMSIW-DGS cell clearer, some investigations on different parameters are discussed in detail. All investigations are carried out under the same conditions of relative permittivity $\varepsilon_{r}=2.2, h=0.254 \mathrm{~mm}, R=0.25 \mathrm{~mm}$, and $D=0.8 \mathrm{~mm}$, by using the same commercial FEM simulator mentioned above. The first investigation is about the influence of the DGS's size, which is mainly determined by the longitudinal length of the slots " $d$ " and the transverse length of the slots " $l$ " in Figure 1(a). Figure 3 shows the transmission response of proposed cell with different " $d$ " and "l." According to the simulated results, as " $d$ " or " $l$ " gets longer, the upper cutoff frequency and bandgap point shift lower, which means that the larger the DGS is, the lower the upper cutoff frequency and bandgap point are. Furthermore, for combinations " $d=1.0 \mathrm{~mm}, l=4.5 \mathrm{~mm}$ " and " $d=$ $0.5 \mathrm{~mm}, l=5.5 \mathrm{~mm}$," there is " $2 d+l=6.5 \mathrm{~mm}$ "; that is, the slots of these two combinations are with the same area. However, the upper cutoff frequency and bandgap point of " $d=1.0 \mathrm{~mm}, l=4.5 \mathrm{~mm}$ " are lower than those of " $d=$ $0.5 \mathrm{~mm}, l=5.5 \mathrm{~mm}$ "; that is, the longitudinal length of slots has stronger influence on the upper cutoff frequency and bandgap point than the transverse length of slots. In components design, through tuning " $d$ " and " $l$," the upper cutoff frequency and bandgap point can be controlled flexibly.

" $b$," the spacing between two slots, as the second important parameter of proposed cell, is investigated, with its results shown in Figure 4. It can be obtained that, as the spacing decreases, the upper cutoff frequency shifts lower slowly, while the bandgap point shifts lower quite fast, which results in a sharper sideband rejection. And the change of the spacing has little influence on the insertion loss.

Figure 5 shows the simulated results of proposed HMSIW-DGS cell with different " $c$," that is, the metallic spacing between the edge of DGS and the magnetic wall of HMSIW. From Figure 5(a), when " $c=0.0 \mathrm{~mm}$," which means that the edge of DGS has met the magnetic wall, the bandwidth of proposed cell is quite narrow, with very sharp rejection slope and wide stopband. Then, once " $c$ " is not equal to zero, the bandwidth of proposed cell increases enormously and rapidly, with the sideband rejection being much weaker. As shown in Figure 5(b), the $3 \mathrm{~dB}$ bandwidth of " $c=0.0 \mathrm{~mm}$ " is about $500 \mathrm{MHz}$; however, as " $c$ " increases to $0.5 \mathrm{~mm}, 1.0 \mathrm{~mm}$, and $1.5 \mathrm{~mm}$, the corresponding bandwidth is increasing to about $4.5 \mathrm{GHz}, 5 \mathrm{GHz}$, and $5.5 \mathrm{GHz}$, respectively. It can also be obtained from Figure 5(a) that as " $c$ " increases, the upper cutoff frequency of proposed cell shifts higher while the bandgap point shifts lower, which means wider bandwidth and sharper sideband rejection. However, the drawback is that the stopband rejection becomes weaker. Another important effect caused by the spacing between the edge of DGS and the magnetic wall is the passband insertion loss. As depicted in Figure 5(b), the insertion loss gets lower as " $c$ " gets larger. For " $c$ " larger than zero, the passband insertion losses are all less than $0.5 \mathrm{~dB}$, much lower than those of " $c=0.5 \mathrm{~mm}$," which is over $1 \mathrm{~dB}$. The reason for this phenomenon is that, for " $c$ " larger than zero, a continuous metallic cover occurs along the magnetic wall, which means that there is a shortest direct metallic 


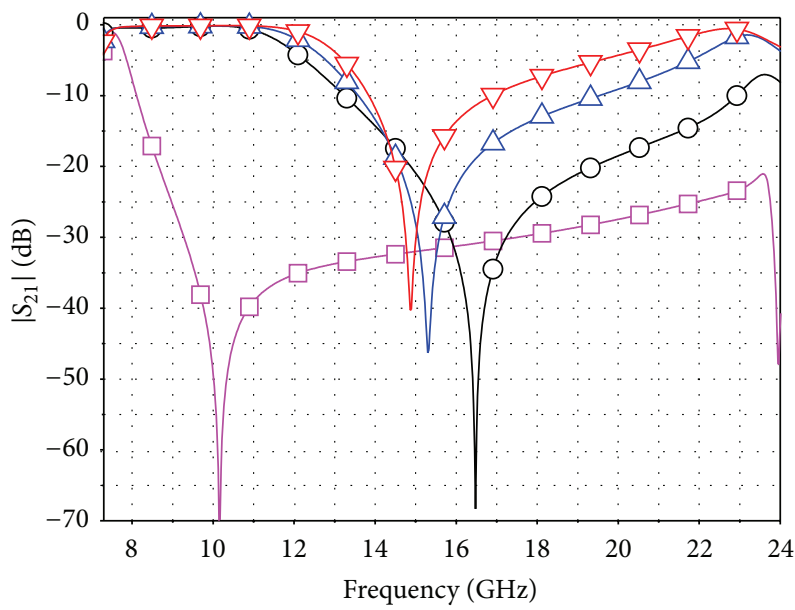

$\begin{array}{rlrl}-\square & =0.0 \mathrm{~mm} & \triangle c & =1.0 \mathrm{~mm} \\ -\bigcirc-c=0.5 \mathrm{~mm} & -\nabla c c & =1.5 \mathrm{~mm}\end{array}$

(a)

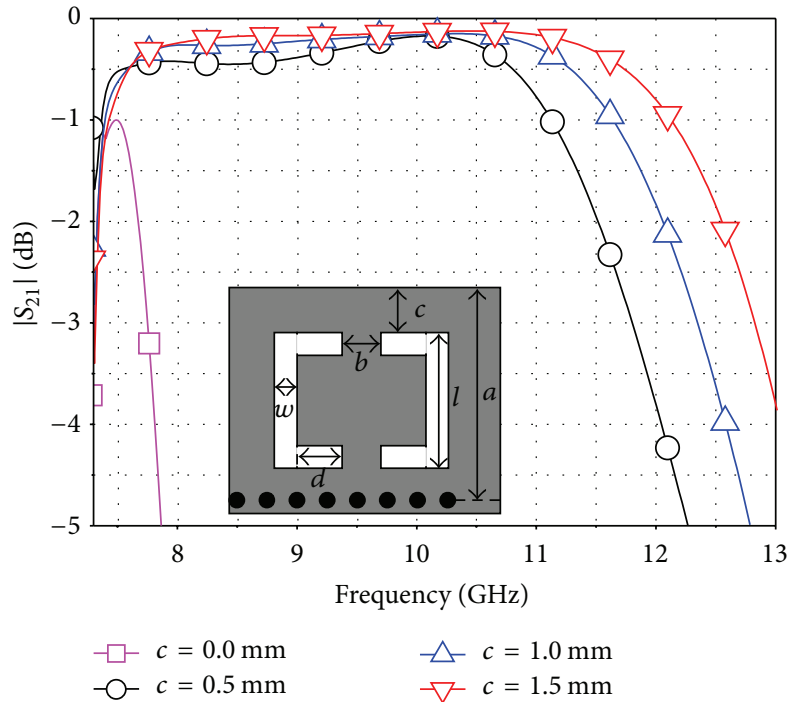

(b)

FIGURE 5: Simulated results of proposed HMSIW-DGS cell with different "c." (a) The whole response and (b) the partial response. Other geometrical parameters are $a=7 \mathrm{~mm}, c=1 \mathrm{~mm}, w=0.5 \mathrm{~mm}, b=1 \mathrm{~mm}$, and $d=1 \mathrm{~mm}$.

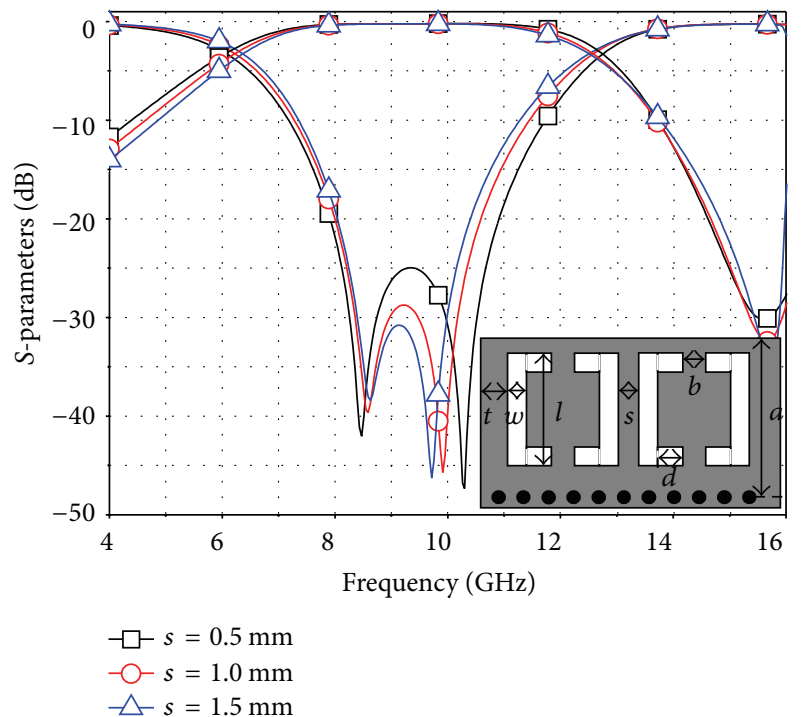

FIGURE 6: Simulated results of intercoupling between two HMSIWDGS cells for varied "s." Other geometrical parameters are $a=$ $7 \mathrm{~mm}, t=1 \mathrm{~mm}, l=5 \mathrm{~mm}, w=0.5 \mathrm{~mm}, d=0.75 \mathrm{~mm}$, and $b=0.5 \mathrm{~mm}$.

path between the input and output ports of the cell. Such a shortest direct metallic path can reduce transmission loss caused by the coupling gaps and provide the best insertion loss performance.

For the embedded form of proposed HMSIW-DGS cell, when the lengths of DGS (" $l 1$," " $d 1$," " $/ 2$," and “ $d 2$ "), the spacing between all couple slots (" $b 1$," " $b 2$ "), and the spacing between the edge of DGS and the magnetic wall (" $c$ ") change, similar effects on the transmission characteristics as the single form can be obtained. Furthermore, the varying of outer slots can cause the cutoff frequency, bandgap point, and stopband rejection change heavily, while the varying of inner slots just brings a little influence on the transmission characteristics. Based on this property, the components design can be more flexible by using outer slots for the coarse tuning and inner slots for the fine tuning.

2.2. Filter Design. Based on the two proposed HMSIW-DGS cells, a three-cell cascaded bandpass filter is designed. There are two essential elements for a cascaded filter design. One is the basic transmission characteristics of the unit cell; the other is the intercoupling between the adjunct cells. For proposed HMSIW-DGS cells, the strength of the intercoupling is mainly controlled by the space between two adjunct cells, as labeled as " $s$ " in Figure 6; the smaller the space is, the stronger the intercoupling is and the wider the separation between the two concave points of the reflection coefficient is. The geometrical configuration of proposed HMSIW-DGS filter, combining three cascaded cells in the middle with two asymmetrical tapered microstrip-HMSIW transitions at input/output ( $\mathrm{I} / \mathrm{O})$ ports, and its optimized dimensions are demonstrated in Figure 7 . The asymmetrical tapered microstrip-HMSIW transitions are utilized to achieve high external quality factor $(Q e)$. $Q e$, as mentioned in [22], can be evaluated by the equation

$$
\mathrm{Q} e=\frac{2 f_{0}}{\mathrm{BW}_{3 \mathrm{~dB}}},
$$

where $f_{0}$ and $\mathrm{BW}_{3 \mathrm{~dB}}$ are the central frequency and $3 \mathrm{~dB}$ rolloff bandwidth of the filter, respectively. In proposed HMSIWDGS filter design, $Q e$ is mainly determined by length and width of the asymmetrical tapered microstrip-HMSIW transitions. For proposed HMSIW-DGS filter shown in Figure 7, 


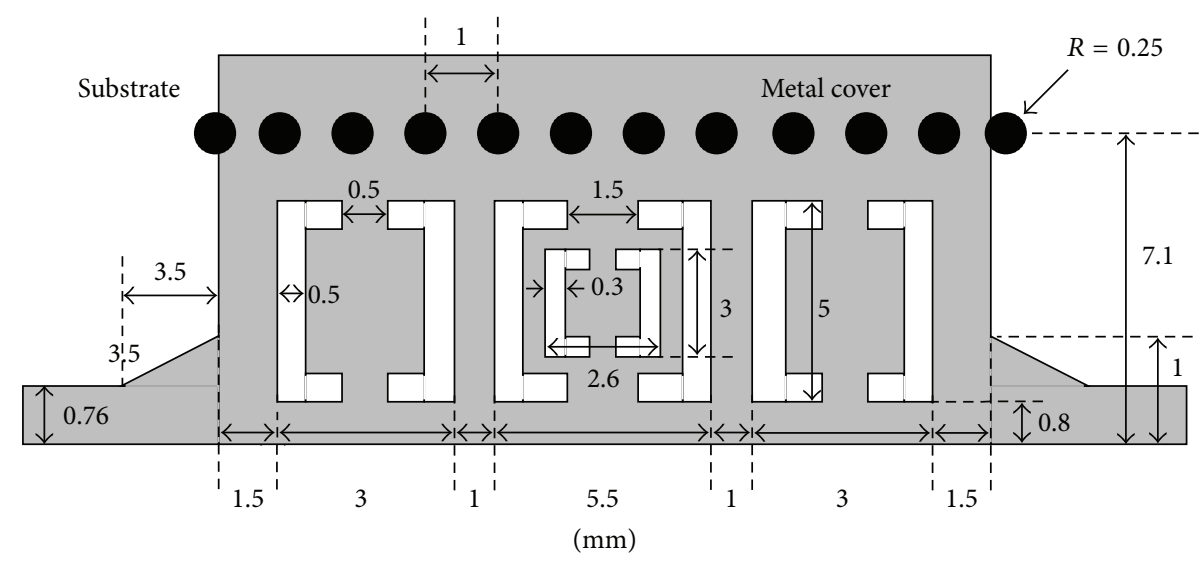

FIGURE 7: Geometrical configuration and dimensions of proposed HMSIW-DGS filter.

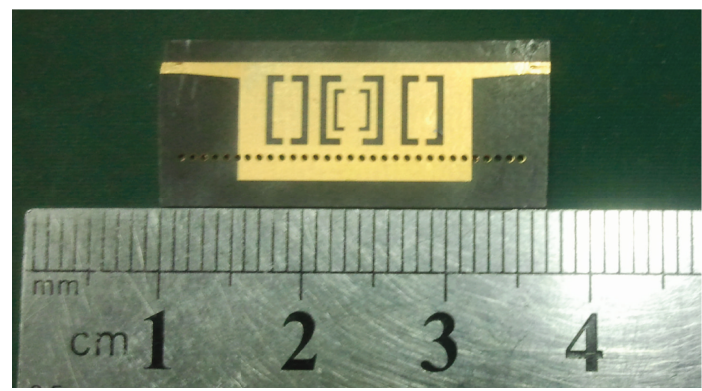

FIGURE 8: Photograph of fabricated HMSIW-DGS filter.

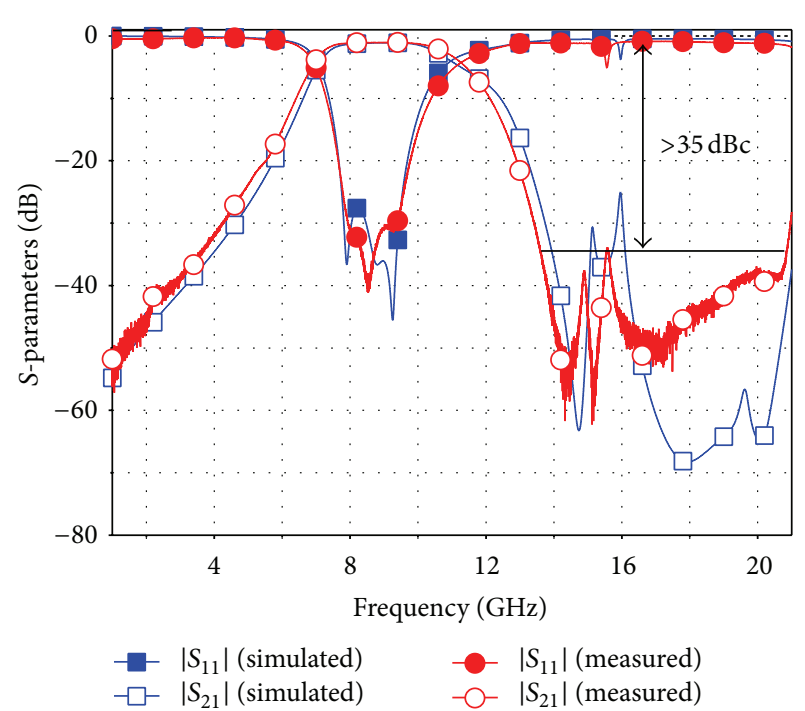

FIGURE 9: Comparison between measured and simulated scattering parameters of proposed HMSW-DGS filter.

its three-dimensional (3D) full wave analyses, including parameters tuning and dimensions optimization, are carried out by using the same commercial FEM simulator mentioned before. And its simulated scattering parameters and group delay are shown in Figures 9 and 10, respectively.

\section{Experimental Results}

The proposed filter, with photograph shown in Figure 8, is fabricated on a $\mathrm{RT} /$ duroid 5880 substrate with relative permittivity of $2.2 \pm 0.02$, loss tangent of 0.001 (at $10 \mathrm{GHz}$ ), and a thickness of $0.254 \mathrm{~mm}$ by standard printed circuits board (PCB) process. This fabricated filter is measured by using an Agilent N5245A vector network analyzer and two $2.4 \mathrm{~mm}$ connectors. According to the measured scattering parameters in Figure 9, the proposed filter achieves a central frequency $\left(f_{0}\right)$ of $8.8 \mathrm{GHz}$, a fractional bandwidth (FBW) of $29 \%$, insertion loss ranging from $0.91 \mathrm{~dB}$ to $1.14 \mathrm{~dB}$, return loss around $30 \mathrm{~dB}$, and second harmonic suppression over $35 \mathrm{~dB}$. Its overall size, including two tapered microstrip-HMSIW transitions, is $27 \times 7.5 \mathrm{~mm}^{2}$, which is quite compact compared with the conventional SIW cavity filters. Figure 10 shows the comparison between simulated and measured group delay of the proposed filter. It can be obtained that the simulated group delay ripple in the passband is around $0.10 \mathrm{~ns}$, while the measured ripple is about $0.15 \mathrm{~ns}$. And the absolute group delay in the passband is less than $0.60 \mathrm{~ns}$. Both the ripple and the absolute group delay show good phase linearity performance, which is quite suitable for wireless data transmission application, especially for the digital microwave communication with high order quadrature amplitude modulation (QAM) [5].

To inspect the performance of proposed filter clearer, some comparisons between proposed filter and previous SIW-DGS or HMSIW-DGS filters reported in the reference are summarized in Table 1. In order to make the comparisons more reasonable, the filters with similar dielectric material, such as relative permittivity of 2.22 and loss tangent of 0.001 (@10 GHz), are selected in references. According to the comparison, filters in $[10,16]$ are with higher insertion loss and no so good return loss. Although filter in [23] has good performance, it is inconvenient to do system integration 
TABLE 1: Comparison with filters presented in references.

\begin{tabular}{|c|c|c|c|c|c|c|c|}
\hline $\begin{array}{l}\text { Reference } \\
\text { filter }\end{array}$ & Order & Topology & $\begin{array}{c}\mathrm{FBW}^{*} \text { and } f_{0}{ }^{*} \\
(\mathrm{GHz})\end{array}$ & Insertion loss $(\mathrm{dB})$ & $\begin{array}{l}\text { Return loss } \\
\quad(\mathrm{dB})\end{array}$ & Size $\lambda_{g}{ }^{2 * *}$ & $\begin{array}{c}\text { 2nd harmonic } \\
\text { suppression }(\mathrm{dBc})\end{array}$ \\
\hline$[10]-1$ & 3 & HMSIW-slot & $22 \%$ and 7.80 & 1.50 & $>10$ & 0.52 & $>20$ \\
\hline$[10]-2$ & 5 & HMSIW-slot & $40 \%$ and 8.79 & 1.20 & $>10$ & 0.37 & $>28$ \\
\hline$[16]$ & 3 & SIW-CSRR & $23 \%$ and 8.15 & 2.16 & 11.6 & 0.64 & \\
\hline [23] & 3 & SIW-CSRR-DGS (bottom) & $30 \%$ and 9.40 & 1.00 & 16 & 1.02 & $>20$ \\
\hline This work & 3 & HMSIW-DGS & $29 \%$ and 8.80 & $0.91-1.14$ & 30 & 0.40 & $>35$ \\
\hline
\end{tabular}

${ }^{*}$ FBW and $f_{0}$ stand for the fractional bandwidth and central frequency of the filters, respectively.

${ }^{* *} \lambda_{g}$ is the guided wavelength of the central frequency of the passband.

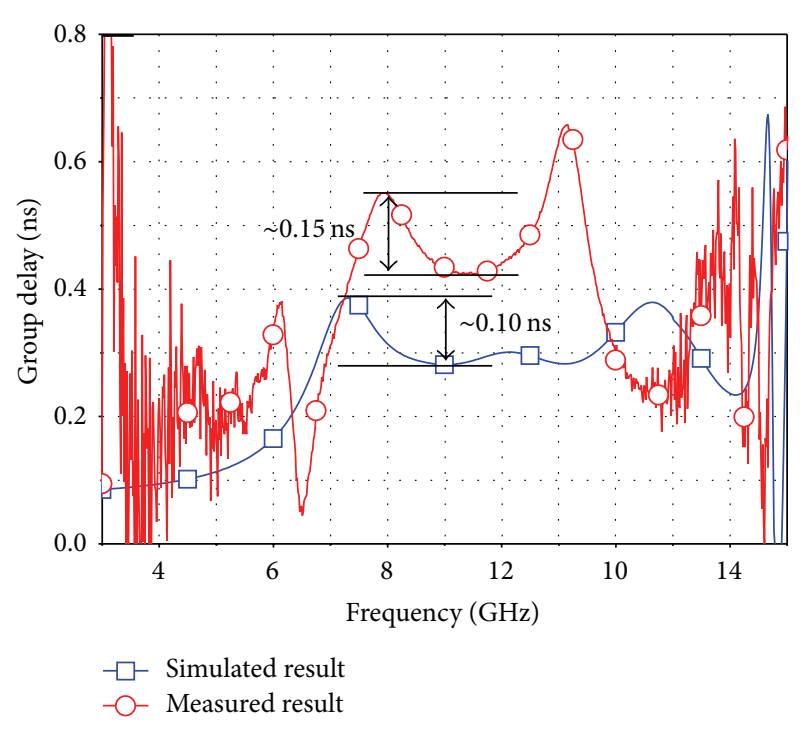

Figure 10: Comparison between measured and simulated group delay of proposed HMSW-DGS filter.

because its DGS is etched into the bottom metal cover. It is obvious that the proposed filter can achieve lower insertion loss, better return loss, more compact size, and better second harmonic suppression.

\section{Conclusion}

An X-band three-cell cascaded bandpass filter based on the two proposed HMSIW-DGS cells is designed, fabricated, and measured. The measured results show good agreement with the simulated one. Compared with some reported filters using the same technology and operating at similar frequency, the proposed filter has better performance in low insertion loss, good return loss, compact size, and second harmonic suppression.

\section{Conflict of Interests}

The authors declare that there is no conflict of interests regarding the publication of this paper.

\section{Acknowledgments}

This work was supported in part by Excellent Ph.D. Candidate Academic Foundation of UESTC under Grant YBXSZC20130154, in part by the Fundamental Research Funds for the Central Universities under Grant ZYGX2013J006, and in part by the Scientific Research Foundation of UESTC under Grant Y02002010101063.

\section{References}

[1] D. Deslandes and K. Wu, "Single-substrate integration technique of planar circuits and waveguide filters," IEEE Transactions on Microwave Theory and Techniques, vol. 51, no. 2, pp. 593-596, 2003.

[2] Y. J. Cheng, H. Xu, D. Ma, J. Wu, L. Wang, and Y. Fan, "Millimeter-wave shaped-beam substrate integrated conformal array antenna," IEEE Transactions on Antennas and Propagation, vol. 61, no. 9, pp. 4558-4566, 2013.

[3] D. Deslandes and K. Wu, "Accurate modeling, wave mechanism, and design consideration of a substrate integrated waveguide," IEEE Transactions on Microwave Theory and Techniques, vol. 54, no. 6, pp. 2516-2526, 2006.

[4] G.-Q. Luo, T.-Y. Wang, and X.-H. Zhang, "Review of low profile substrate integrated waveguide cavity backed antennas," International Journal of Antennas and Propagation, vol. 2013, Article ID 746920, 7 pages, 2013.

[5] Y.-M. Huang, Z.-H. Shao, and L.-F. Liu, "A substrate integrated waveguide bandpass filter using novel defected ground structure shape," Progress in Electromagnetics Research, vol. 135, pp. 201-213, 2013.

[6] G. Zhai, Y. Cheng, Q. Yin, L. Chiu, S. Zhu, and J. Gao, "Super high gain substrate integrated clamped-mode printed logperiodic dipole array antenna," IEEE Transactions on Antennas and Propagation, vol. 61, no. 6, pp. 3009-3016, 2013.

[7] H. Vettikalladi and M. A. S. Alkanhal, "BCB-Si based wide band millimeter wave antenna fed by substrate integrated waveguide," International Journal of Antennas and Propagation, vol. 2013, Article ID 816050, 4 pages, 2013.

[8] W. Hong, B. Liu, Y. Wang et al., "Half mode substrate integrated waveguide: a new guided wave structure for microwave and millimeter wave application," in Proceedings of the 31st International Conference on Infrared and Millimeter Waves and 14th International Conference on Terahertz Electronics, p. 219, Shanghai, China, September 2006.

[9] B. Liu, W. Hong, Y.-Q. Wang, Q.-H. Lai, and K. Wu, "Half mode substrate integrated waveguide (HMSIW) 3-dB coupler," IEEE 
Microwave and Wireless Components Letters, vol. 17, no. 1, pp. 22-24, 2007.

[10] Y. Wang, W. Hong, Y. Dong et al., "Half mode substrate integrated waveguide (HMSIW) bandpass filter," IEEE Microwave and Wireless Components Letters, vol. 17, no. 4, pp. 265-267, 2007.

[11] D. Ahn, J.-S. Park, C.-S. Kim, J. Kim, Y.-X. Qian, and T. Itoh, “A design of the low-pass filter using the novel microstrip defected ground structure," IEEE Transactions on Microwave Theory and Techniques, vol. 49, no. 1, pp. 86-93, 2001.

[12] A. B. Abdel-Rahman, A. K. Verma, A. Boutejdar, and A. S. Omar, "Control of bandstop response of Hi-Lo microstrip lowpass filter using slot in ground plane," IEEE Transactions on Microwave Theory and Techniques, vol. 52, no. 3, pp. 1008-1013, 2004.

[13] A. Abdel-Rahman, A. R. Ali, S. Amari, and A. S. Omar, "Compact bandpass filters using Defected Ground Structure (DGS) coupled resonators," in Proceedings of the IEEE MTT-S International Microwave Symposium, pp. 1479-1482, June 2005.

[14] J. Lim, C. Kim, D. Ahn, Y. Jeong, and S. Nam, "Design of lowpass filters using defected ground structure," IEEE Transactions on Microwave Theory and Techniques, vol. 53, no. 8, pp. 25392544, 2005.

[15] Z.-H. Shao and M. Fujise, "Bandpass filter design based on LTCC and DGS," in Proceedings of the Asia-Pacific Microwave Conference (APMC '05), vol. 1, pp. 138-139, December 2005.

[16] X.-C. Zhang, Z.-Y. Yu, and J. Xu, "Novel band-pass substrate integrated waveguide (SIW) filter based on complementary split ring resonators (CSRR)," Progress in Electromagnetics Research, vol. 72, pp. 39-46, 2007.

[17] F. Falcone, T. Lopetegi, J. D. Baena, R. Marqués, F. Martín, and M. Sorolla, "Effective negative- $\varepsilon$ stopband microstrip lines based on complementary split ring resonators," IEEE Microwave and Wireless Components Letters, vol. 14, no. 6, pp. 280-282, 2004.

[18] Y. D. Dong, T. Yang, and T. Itoh, "Substrate integrated waveguide loaded by complementary split-ring resonators and its applications to miniaturized waveguide filters," IEEE Transactions on Microwave Theory and Techniques, vol. 57, no. 9, pp. 2211-2223, 2009.

[19] L. Zhou, S. Liu, N. Gao, Y. Chen, and Y. Wei, "Miniaturized substrate integrated waveguide filter with complementary spiral resonator," Microwave and Optical Technology Letters, vol. 53, no. 6, pp. 1308-1311, 2011.

[20] F. Xu and K. Wu, "Guided-wave and leakage characteristics of substrate integrated waveguide," IEEE Transactions on Microwave Theory and Techniques, vol. 53, no. 1, pp. 66-73, 2005.

[21] Q. Lai, C. Fumeaux, W. Hong, and R. Vahldieck, "Characterization of the propagation properties of the half-mode substrate integrated waveguide," IEEE Transactions on Microwave Theory and Techniques, vol. 57, no. 8, pp. 1996-2004, 2009.

[22] J. S. Hong and M. J. Lancaster, Microstrip Filter for RF/Microwave Applications, chapter 8, John Wiley \& Sons, New York, NY, USA, 2001.

[23] K. Deng, Z. Guo, C. Li, and W. Che, "A compact planar bandpass filter with wide out-of-band rejection implemented by substrate-integrated waveguide and complementary split-ring resonator," Microwave and Optical Technology Letters, vol. 53, no. 7, pp. 1483-1487, 2011. 

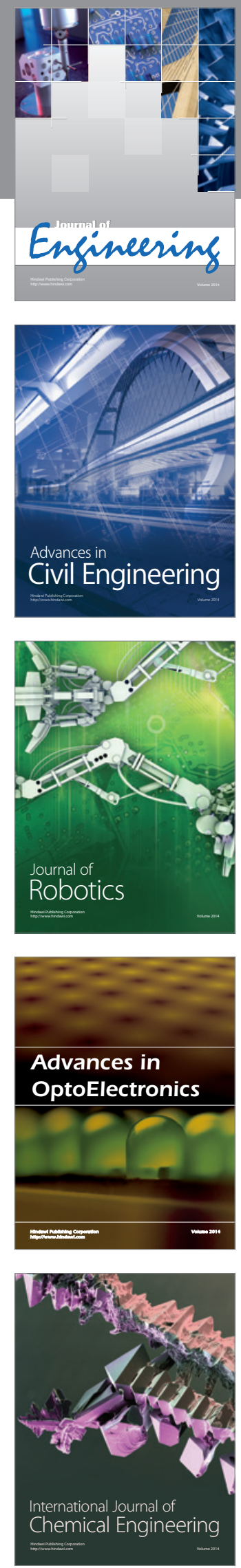

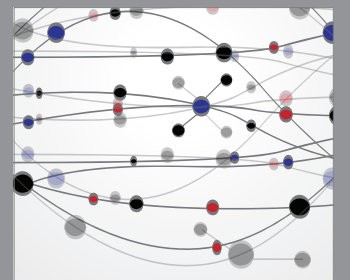

The Scientific World Journal
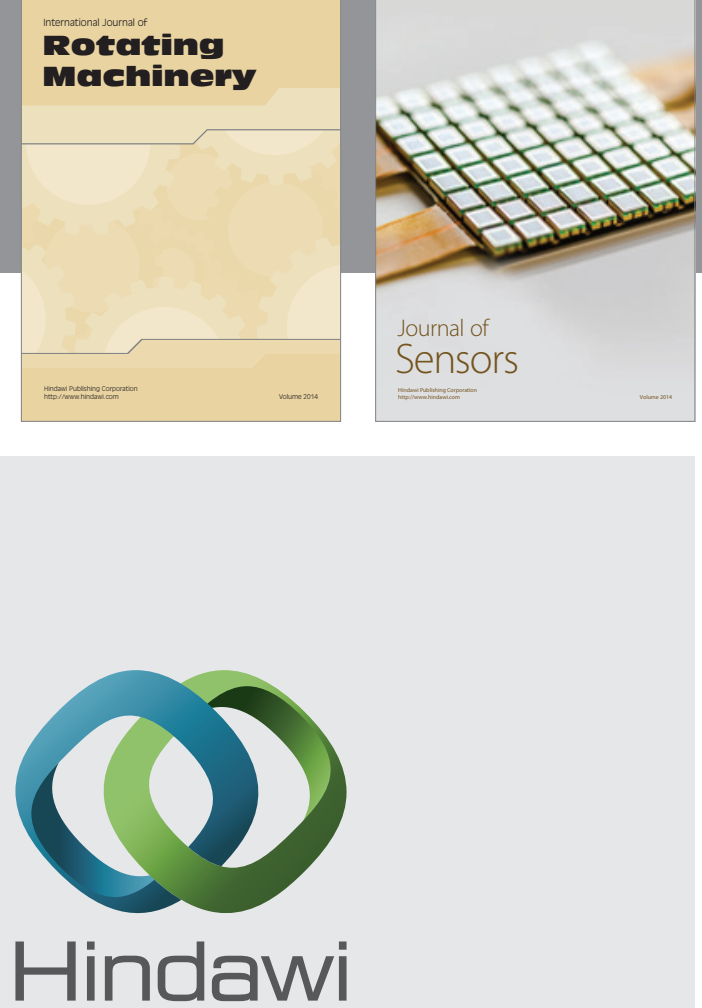

Submit your manuscripts at http://www.hindawi.com
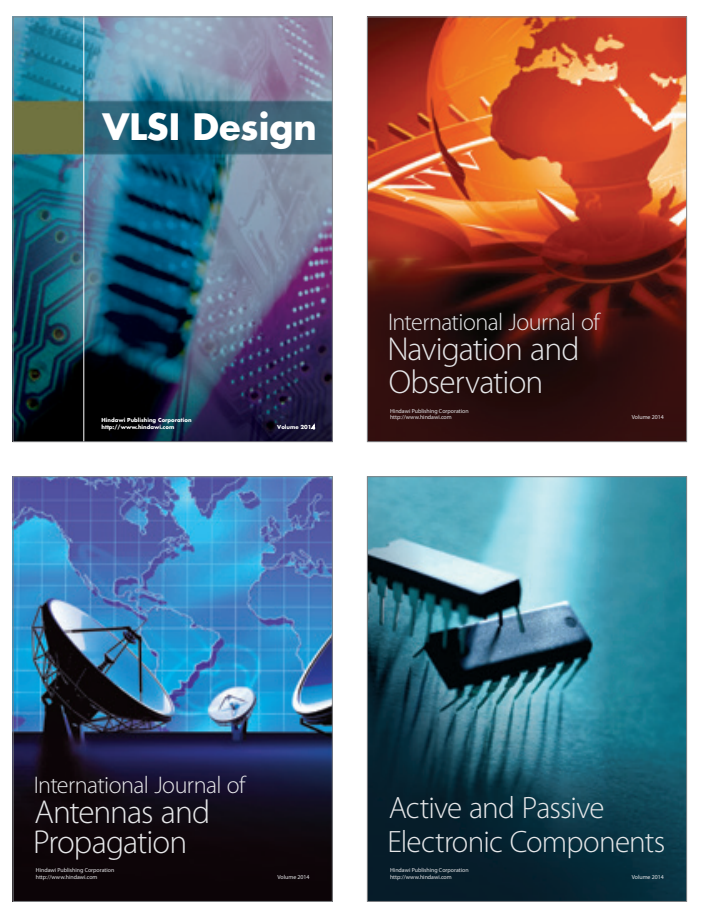
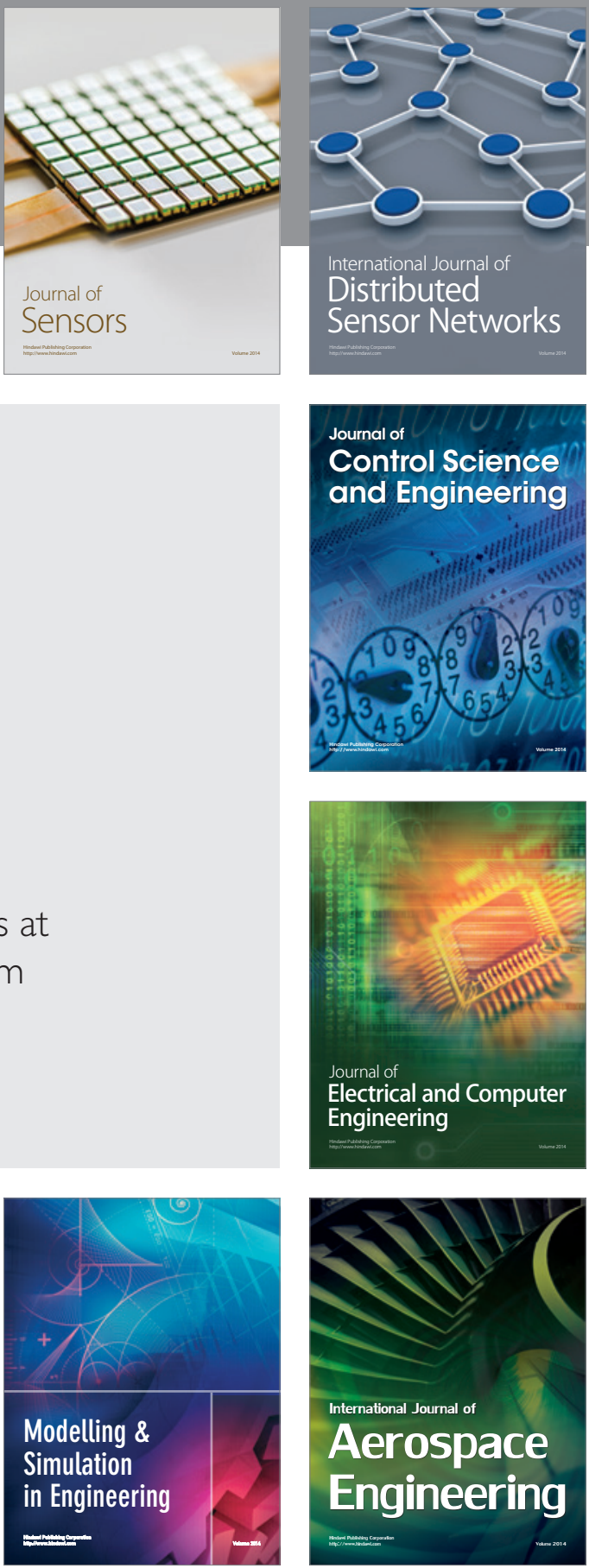

Journal of

Control Science

and Engineering
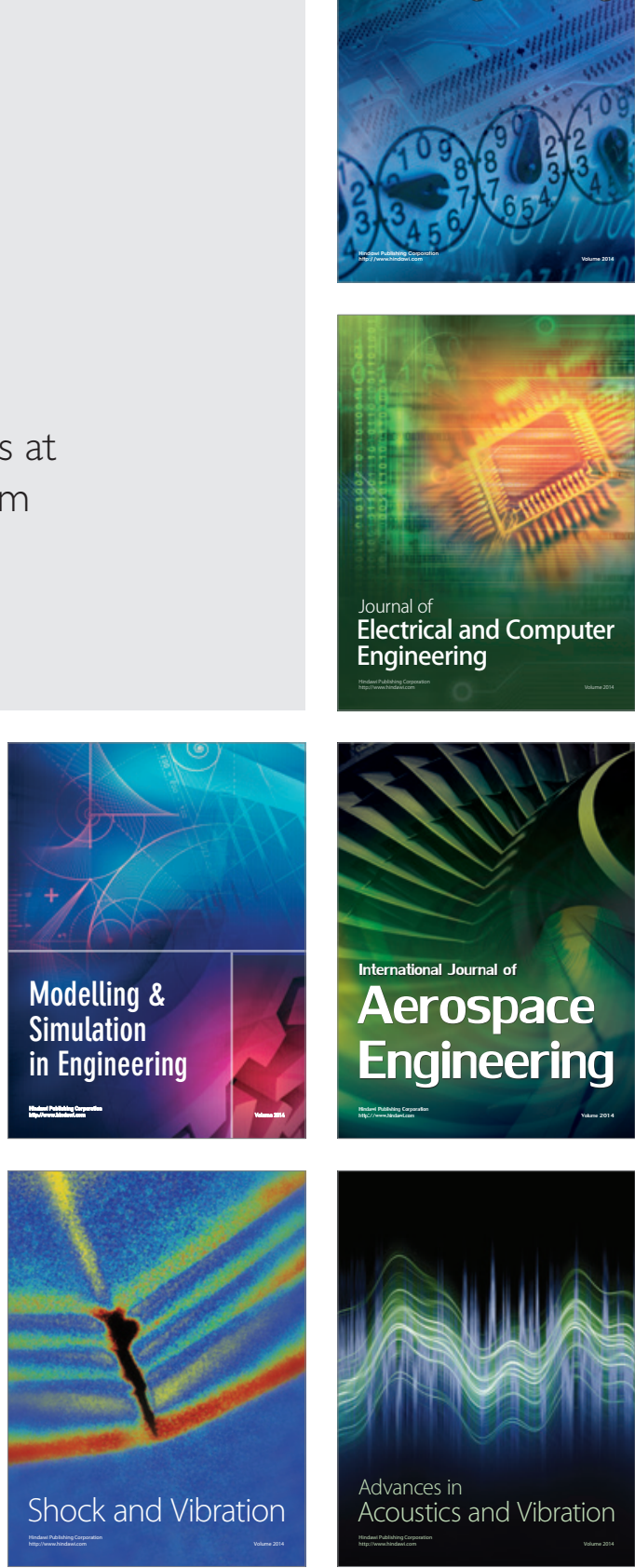